\title{
Estrés y el burnout como riesgos en los escenarios laborales después de la emergencia: conceptualizaciones y ejes de análisis para la gestión preventiva en las organizaciones
}

\author{
Stress and burnout as risks in the labor place after the \\ emergency: conceptualizations and axes of analysis for \\ the preventive management in organizations
}

\section{KaRIN RoA}

Karin Roa es psicóloga UC. Magíster en Gobierno de Organizaciones, Universidad de Navarra, España. Actualmente es docente de la Escuela de Trabajo Social PUC y miembro asociado del Centro de Estudios de Emprendimientos Solidarios, CEES-UC. Su dirección electrónica es kroa@uc.cl, su dirección postal es Av.Vicuña Mackenna 4860, Macul - Campus San Joaquín.

\begin{abstract}
Resumen
Este artículo examinar las bases psicosociales del estrés y el burnout en el trabajo, enfatizando el papel que en su origen o contención juegan las organizaciones, entendidas como sistemas sociales que ofrecen a las personas escenarios para el desempeño de roles, identidades, así como de significación personal. Todo lo anterior, en busca de argumentaciones que ayuden a comprender el mundo del trabajo no sólo como un escenario más para la vivencia de crisis y desastre, sino también como un lugar que puede afrecer oportunidades para la recuperación y el desarrollo personal de los trabajadores.

Palabras claves. Estrés ocupacional y burnout; organizaciones, trabajo, desastre.

Abstract

This article studies the psychosocial bases of stress and burnout field of labor, emphasizing the role organizations play in either, starting or containing them understanding organizations as social systems offering scenarios for roles and identities performance, as well as personal signification. This reflection is carried aut in search for arguments helping to understand the field of labor not only as another scenario where to experience crisis and disaster, but also as a place that can provide opportunities for employees' recovery and personal development.
\end{abstract}

Keywords. Occupational Stress and-out, organizations, work, disastres.

\section{Introducción}

Una idea que ha sido explorada desde la antiguedad en la psicología laboral es que en la relación entre trabajador y trabajo la influencia es mutua: las personas condicionan el cómo un puesto se desempeña y así también el puesto de trabajo y la organización tienen impacto en la subjetividad e identidad de los trabajadores. Esta es una de las vías por la que se ha explicado el impacto del trabajo en la salud mental de las personas.

El objetivo de este artículo es revisar algunos con- ceptos e ideas que permitan comprender cómo el trabajo en condiciones precarias tras un desastre natural pueden constituirse en factores que predispongan el desarrollo de dos enfermedades ocupacionales: el estrés y el burnout, ambas de comprobado impacto negativo en la calidad de vida del trabajador y también en la efectividad de las organizaciones.

Con miras a ese objetivo se revisarán en primer lugar las definiciones de estrés y burnout, haciendo énfasis en perspectivas psicosociales y modelos 
transaccionales que definen estas enfermedades como fruto de experiencias que socavan el sentido de lo que se aporte y, más grave aún, la valía de quien se es.

Luego se revisarán cuatro aspectos que, se sugiere, pueden ser observados y administrados en la gestión de las organizaciones de ayuda y primera respuesta tras meses del terremoto, como forma de prevenir efectos patológicos: (1) la situación de crisis general como contexto sobre el que se desarrolla el trabajo (2) Factores psicosociales que se presentan en el nuevo contexto laboral asociados a estrés y burnout; (3) Sobrecargas en el trabajo emocional; y (4) Modificaciones en las condiciones y recompensas habituales de la relación de ayuda.

Finalmente, se sugieren algunos aspectos generales que deben ser tomados en cuenta en la gestión organizacional con el fin de promover el trabajo como un espacio preventivo y promotor de desarrollo personal y organizacional en condiciones de crisis y emergencia prolongada, como la que se observa aún hoy en el país.

\section{Estrés: conceptualización desde los modelos transaccionales}

Bajo la influencia de la perspectiva psicosocial y de la teoría del aprendizaje social en psicología, el estrés se ha entendido como respuesta a una relación perjudicial de sobredemanda hacia la persona respecto de los arreglos organizacionales, del ambiente físico y relaciones interpersonales en el trabajo; ello condiciona negativamente la manera en que los trabajadores evalúan su experiencia, el cómo piensan, sienten y actúan, traduciéndose en efectos negativos para los equipos y organizaciones (Gil-Monte y Peiró, 1997; Párraga, 2005). Estas son las premisas que dan lugar a los llamados modelos transaccionales en salud mental ocupacional (Cox, Griffiths y RialGonzález, 2005).

Este modelo, originalmente planteado por Karasek, continuado luego por Lazarus y Folkman en Michigan y actualmente fortalecido por Tom Cox y su equipo desde Nottingham, parte de la premisa que no hay estresores "en sî" o situaciones estresantes objetivamente hablando; se trata más bien de la constitución de una experiencia con tres rasgos: (a) la persona percibe su incapacidad para encarar las demandas del entorno o las amenazas a su bienestar; (b) el responder ante la situación es relevante para la persona; (c) se producen cambios físicos, cognitivos y emocionales de los que se es conciente, caracterizados por un estado de ansiedad o depresión, inquietud, insomnio o palpitaciones y pérdida gradual de la concentración y la creatividad, de la confianza y de tomar decisiones, todo lo cual constituye lo que normalmente las personas describen como "sentirse estresado"( Cox, Griffiths y RialGonzález, 2005; Parra, 2003).

Como se ve, esta forma de ver el estrés permite explicar cómo ciertas condiciones que parecerían extremas a un observador no resultan estresantes para los trabajadores que las confrontan con éxito; o estresan a algunos, pero no a todos, por lo que el análisis de las transacciones entre persona y entorno es la clave para comprender el origen y evolución de un cuadro como este en las particulares circunstancias de crisis post-terremoto.

Respecto de los factores que lo originan, el estrés se interpreta como producto de la percepción de intercambios desbalanceados entre las presiones del contexto y las estrategias y aprendizajes previos que la persona despliega frente a esos factores (Bryce, 2001; Cohen, 1998; Cox, Griffiths y Rial-González, 2005; Trucco, Valenzuela y Trucco, 1999). A esto se llama "estrategias de afrontamiento"(Cox, Griffiths y Rial-González, 2005; López-Cabañas y Chacón, 1999). Si una estrategia resulta efectiva, por ejemplo buscar ayuda de otros para cumplir tareas en un plazo anormalmente breve, la persona se siente nuevamente en una situación de capacidad y ajuste entre lo que puede dar y las demandas: cumple y experimenta la satisfacción por haber cumplido. Si el afrontamiento no resulta exitoso, deviene la experiencia de estar sobrepasado porque los esfuerzos resultan infructuosos.

Por ejemplo, una de las estrategias de afrontamiento que se han estudiado frente al estrés agudo en personas con roles de ayuda es la despersonalización. Ésta se entiende como un aumento de la distancia psicológica con el usuario, que puede traducirse en un continuo desde la leve indiferencia hasta un extremo de relación deshumanizante con el otro, que tiene por fin el minimizar el sufrimiento que produce el contacto poco recompensante con ellos o la impotencia de no poder ayudarles (Freudenberger, 1974; Maslach, 1981; Gil-Monte, 1999).

En las ocupaciones donde la capacidad del contacto personal cercano y deferente es relevante, un enfriamiento afectivo respecto del usuario se traduce en una reducción en la calidad de la atención que se les ofrece, toda vez que la empatía permite contactarse y así comprender mejor a las personas y lo que buscan. Además ese trato cercano suele ser fuente de satisfacción y sentido del trabajo para personas con vocación de servicio, sentido que se esfuma cuando se responde desde la despersonalización. Adicional- 
mente, es normal en las organizaciones de ayuda que se tenga el trato empático dentro de las expectativas de rol, por lo que la frialdad se sanciona social o concretamente por jefaturas o compañeros.

Desde todo punto de vista en este caso hipotético el afrontamiento resulta infructuoso, por lo que se eleva la experiencia de estrés agudo, en vez de disminuir: no se resuelven satisfactoriamente las exigencias del puesto. Si las condiciones de trabajo se mantienen constantes, es posible que el estrés agudo se vuelve permanente y aumenta el riesgo de evolucionar hacia una enfermedad ocupacional crónica de creciente popularidad: el burnout.

\section{Del estrés al burnout: los riesgos del riesgo prolongado}

Especialmente vinculada y estudiada en profesiones y ocupaciones de ayuda y salud como psicología, trabajo social, enfermería y profesorado, el burnout se define como un proceso de desilusión progresiva en el trabajo, que contrasta con un momento primero en que éste producía altos niveles de motivación, involucramiento e identificación emocional (Gil-Monte y Peiró, 1999; Gil-Monte, 2003; Freudenberger, 1974; Maslach, 1981, 2001, 2009; Pines en Schaufeli, Maslasch y Marek, 1993).

Así como el estrés se define como la experiencia de sentirse sobredemandado(a) e impotente para afrontar efectivamente el contexto, el burnout se refiere a un proceso por el que tras vivir estrés agudo por períodos relativamente prolongados, el trabajo deja de aportar satisfacciones de tipo intrínseco, pierde sentido y produce sentimientos de desvalorización del puesto, del sí mismo, culpa y finalmente las consecuencias negativas más mencionadas: morbilidad psiquiátrica, interferencia con las actividades productivas y enfermedades somáticas gatilladas por factores psicológicos (Parra, 2001).

Los síntomas más frecuentemente descritos en la literatura, y en su orden de aparición, son los siguientes:

- Agotamiento emocional. Se refiere a una situación de agotamiento de la capacidad o de los recursos emocionales propios, una experiencia de estar emocionalmente agotado debido al contacto intenso y mantenido con personas a las que hay que atender. Se entiende entonces la centralidad del factor de riesgo en que se transforma el trabajo emocional en ocupaciones de ayuda, y más aún, en situaciones de emergencia (Gil-Montes y Peiró, 1997; Gil-Monte, 2003; Maslach, 1981, 2001, 2009)
- Baja percepción de logro o realización personal en el trabajo. Se refiere a la tendencia de los trabajadores en burnout a evaluarse negativamente en torno a la habilidad en la realización del trabajo y a la relación con los usuarios. Los trabajadores se sienten descontentos consigo mismo e insatisfechos con sus resultados laborales (Gil-Monte y Peiró, 1997; Gil-Monte, 2003; Maslach, 1981, 2001, 2009).

- Despersonalización. Se refiere al desarrollo de actitudes y sentimientos negativos, como el cinismo, hacia las personas destinatarias del trabajo. Dado un endurecimiento afectivo, se pierde la perspectiva de vulnerabilidad y se deshumaniza la forma en que el trabajador ve al usuario. Puede manifestarse en un trato duro, indiferente, el poner sobrenombres o motes para clasificar o identificar a las personas, y en el extremos más disfuncional, el trato humillante o vejatorio (Gil-Monte, 2009). Se le considera una forma de afrontamiento más bien tardía y como una respuesta para afrontar el estrés que las interacciones con estos usuarios gatilla (Gil-Montes y Peiró, 1997; Gil-Monte, 2003; Maslach, 1981, 2001, 2009).

- Culpa. Es el último de los síntomas en aparecer y se entiende como correlato ante la sensación de pérdida de contacto con los usuarios y de la calidad en los aportes, en contextos en que la expectativa de rol tiene que ver con el cuidado, la calidez, la vocación de servicio. Se ha observado que como modo de eludir o reducir la culpa se responde con un afrontamiento consistente en aumentos de horas y de compromiso, que si resultan infructuosos, agudizan los tres primeros síntomas, perjudicando y profundizando la evolución del cuadro (Gil-Montes y Peiró, 1997; Gil-Monte, 2009).

Como efectos negativos a nivel organizacional, se ha estudiado que el burnout socava el buen desempeño y la calidad del servicio ofrecido por las personas que lo padecen y concomitantemente el desempeño del grupo; produce insatisfacción y déficit en el clima laboral; aumenta el ausentismo; deterioran las relaciones interpersonales y aumenta la disposición de renunciar el puesto de trabajo incrementando las tazas de rotación de personal (Freudenberger, 1974; Gil-Monte y Peiró, 1999; Gil-Monte, 2007; Wright y Copranzano, 1998; Pines, 1993 en Schaufeli, Maslasch y Marek, 1993; Maslach, Schaufeli y Leiter, 2001; Maslach, 2009). Estudios realizado en contextos profesionales específicos muestran por 
ejemplo que las enfermeras que experimentan niveles más elevados de burnout proporcionaban, según los pacientes, niveles más bajos de cuidado (Leiter et al. 1998 citado en Maslach 2009), mientras que otro estudio descubrió que policías con altos niveles de burnout reportaban más uso de violencia en contra de la población (Kop et al. 1999 en Maslach, 2009).

Habiendo explicitado las definiciones y desarrollos actuales en referencia al estrés y al burnout, se ofrece a continuación una caracterización de factores del entorno laboral con los que pueden interactuar los trabajadores en situaciones de crisis por desastre natural, y que han demostrado predisponer experiencias negativas en relación el entorno laboral de los profesionales de ayuda.

\section{Los desastres naturales como escenarios de crisis}

Desde la perspectiva de la salud mental, los desastres son fuentes de riesgo de estrés puesto que se viven como crisis vitales que alteran la vida cotidiana y que exceden la capacidad habitual de las personas para enfrentar problemas (Caplan, 1964). En términos del impacto psicosocial, es común que entre un tercio y la mitad de la población afectada sufra manifestaciones psicológicas, como producto de una sobredemanda en el ámbito individual, familiar y social (Organización Panamericana de la Salud (OPS), 2002, 2006). Entre las reacciones más frecuentes tras estos desastres están la ansiedad, la depresión y en casos algo más extremos, el síndrome de estrés post-traumático (OPS, 2002).

Sin embargo, no por eso toda crisis se asocia con resultado patológico. Si las personas afectadas son capaces de encontrar, y el entorno es capaz de proveer, condiciones para construir sentimientos de confianza y procesos de defensa, maduración y adaptación, la crisis será un mal recuerdo, pero un recuerdo integrado sanamente a la biografía. Sin embargo, cuando las posibilidades de adaptación se ven sobrepasadas por los hechos, por la cuantía del deterioro físico, social o laboral, o por condiciones de vida precarizadas previamente, es posible que esas crisis pasen a constituir eventos traumáticos y estresantes y devenga de ello la experiencia de sufrimiento psicológico (OPS, 2006).

Un factor que puede implicar riesgos adicionales de resultados negativos en la salud mental de la población es que la crisis se prolongue (OPS, 2006). En el caso de Chile, las réplicas tras el terremoto del 27 de Febrero se sucedieron hasta sesenta días después del terremoto, a razón de hasta cuatro por jornada, tanto de día como de noche, manteniendo vigente el estado de incertidumbre y alerta en la población. Ello supuso además un incremento continuo del deterioro de la infraestructura, las dificultades de obtener ayuda y la desmoralización concomitante. Por otro lado, y asociado a la llegada del invierno, existe la posibilidad que para algunos grupos más afectados la crisis adquiera nuevo dramatismo, reflejadas en vulnerabilidades derivadas de la desprotección en vivienda, del surgimiento de enfermedades, etc.

Entendida como un factor de riesgo de estrés, las crisis prolongadas desafían la capacidad de recuperación de las personas, demoran la elaboración de un nuevo estado de equilibrio y por ende exponen a la población a mayor riesgo de percepción de sobredemanda, que se sumana la necesidad de tener que seguir funcionando en aspectos básicos de la vida cotidiana. Como señales de ésta experiencia, es común el surgimiento de sentimientos de impotencia, desesperanza y agotamiento emocional, ansiedad y miedo, acompañados de un deterioro en la capacidad de reflexión y de toma de decisiones, que se vuelven características del funcionamiento en este estado de sobredemanda prolongada (OPS, 2006).

Adicionalmente, y a diferencia de las crisis individuales, las crisis derivadas de desastres naturales imponen un escenario de apoyo y soporte social cualitativamente diferente. En situaciones de crisis individuales, las personas suelen contar con quienes les rodean para obtener apoyo, consejo, compañía, etc.. Sin embargo, tras un evento que afecta colectivamente a la población, es frecuente que se desorganicen o deterioren gravemente las redes sociales, toda vez que los miembros están afectados e impotentes frente a la situación de desastre y con mayor propensión a labilidad y conflictos interpersonales (OPS, 2006). Así, ésta es una crisis más solitaria que otras de corte personal, razón por la que las que ciertas estrategias de afrontamiento que se basan en la familia o la comunidad, en otras ocasiones disponibles, no funcionan.

De la confluencia de estos factores se desprende la explicación del por qué aproximadamente un tercio de las personas, una vez transcurridos hasta seis meses del evento, no logran alcanzar un nuevo estadio saludable, evidenciando los efectos más estables y menos deseables de patología psicológica asociada. Es por ello que una respuesta efectiva para el manejo de la salud mental de la población debe extenderse por al menos un semestre, y preparar respuestas adecuadas para este momento.

Esta descripción del estado de crisis general tras un 
desastre natural se incluye como factor de riesgo para el estrés y burnout laboral porque en su mayoría las personas que se abocan a la ayuda social y de salud son parte de la población afectada. De hecho, se suele recomendar que la primera respuesta y los grupos de ayuda sean miembros de la comunidad, que conozcan el lugar, situaciones y aspectos culturales locales (OPS, 2002, 2006).

El riesgo para estos trabajadores emerge precisamente del hecho que pueden iniciar labores de rescate o apoyo tras el desastre en condiciones en que comparten la misma situación subjetiva de crisis o duelo que el resto de la población, sus consecuencias, sus precarizaciones y los riesgos de enfermar. De allí que su capacidad de trabajar, de sobreponerse al efecto de estresores adicionales y específicos del entorno laboral (que a continuación se señalan) y de contener el sufrimiento de otros, puede verse debilitada tanto a corto, pero más preocupante aún, a largo plazo.

\section{Factores psicosociales de riesgo para los tra- bajadores}

Los factores psicosociales se definen como aspectos presentes en una situación laboral, relacionadas con el diseño y la organización del trabajo, con el contenido del puesto, con la realización de la tarea e incluso con el medio físico, que tienen el potencial de afectar al desarrollo del trabajo y de causar daño a la salud física, social o psicológica de los trabajadores (Cox, Griffiths y Rial-González, 2005; Gil-Monte, 2009).

Tras el terremoto el escenario laboral en ciertas instituciones principalmente de ayuda, salud y seguridad pública puede haber cambiado para los trabajadores. A continuación se destacan algunos factores que tienen potencial de superar las capacidades de adaptación de los trabajadores y que, en algunos casos, persisten aún por meses tras ocurrido el evento crítico (extraído de Bryce, 2001; Cohen, 1998; OPS, 2002, 2006):

- La obligación de mantenerse alejados de la familia por razones laborales, o desinformado del paradero de parientes o amigos. Puede ocasionar tensiones y recriminaciones por parte de familiares dado que el trabajador se ausentan en momentos de mucha incertidumbre, temen por su seguridad o necesitan de su ayuda (OPS, 2006).

- Demandas de gran esfuerzo físico y mental, a veces sin soporte adecuado de agua y alimentos, sobreesfuerzos de largas jornadas sin descanso o de trabajo nocturno, no usuales (Bryce, 2001; OPS, 2002)

- Deterioro del ambiente físico del trabajo. Inmediatamente después del terremoto algunos profesionales de ayuda debieron comenzar a trabajar en condiciones precarias. Numerosos son los testimonios del trabajo en plazas, en carpas, en dependencias seguras pero ajenas, en ausencia de condiciones mínimas: sin su computador, fichas o carpetas, o lamentando la pérdida de información valiosa e irrecuperable, sin baño, sin lugar donde comer o con escases de agua.

En el corto plazo, estas condiciones de trabajo pueden aceptarse como parte del anecdotario de actos sacrificados que vale la pena atesorar en los anales de la organización. En el mediano plazo, la falta de medios incomoda, e incluso impide el normal desarrollo de las funciones asignadas, pese a que las presiones por resultados no desaparecen proporcionalmente. En el largo plazo, esas nuevas condiciones pueden vivirse como poco tolerables, pudiendo interpretarse como una falta de estatus social en el trabajo. Alejados de condiciones de trabajo dignificantes, el riesgo allí donde persistan es que las personas terminan concluyendo que su trabajo, y ellos mismos, no son importantes, que no tienen relevancia.

- Sobrecarga de trabajo. Necesidad de cumplir con muchas tareas en forma rápida y pese a la falta de materiales, tiempo o información del puesto de trabajo. Se experimenta como la sensación de sobrecarga y de desequilibrio entre lo disponible y lo solicitado (OPS, 2002). La sobredemanda puede ser resuelta invirtiendo tiempo adicional y quitando tiempo a otros dominios de la vida, como la familia, con el consiguiente trastorno, cansancio e insatisfacción asociada.

- Pérdida de control y certidumbre. Asociado a la sobrecarga, se manifiesta como sensación de haber perdido la capacidad de controlar aquello por lo cual se les está responsabilizando. Asimismo la falta de control puede devenir de los cambios en la organización o tipo de trabajo que deben desarrollar (Maslach, 2009)

- Problemas en las dinámicas internas de equipo en torno a comunicación, solución de problemas, toma de decisiones, conflictos interpersonales, etc. (Maslach, 2009). Así, el contacto con compañeros y jefaturas se vuelve un factor adicional de tensiones e insatisfacciones, a la vez que obstaculiza el logro de metas. 
- Interferencias en las funciones. Propio de situaciones en que es necesario coordinarse o trabajar en equipo con personal con el que no se suele colaborar o con quienes se rivaliza en estilos o metas de trabajo (OPS, 2002). La llegada de voluntarios, personas de gobierno central, cuerpos de apoyo o medios de comunicación demanda adecuarse y desempeñarse con más observadores y más interacciones de lo usual y quizá en funciones nuevas, todo lo cual puede encontrar al trabajador poco preparado para hacer frente a ese desafío.

- Trabajo expuesto a los medios de comunicación. Tanto la operación como los resultados o las quejas de los usuarios aparecen exponiendo a los trabajadores y unidades de apoyo, principalmente en torno a quejas, demoras o fracasos de las gestiones (OPS, 2006)

- Incomodidad en las funciones. Un aspecto importante, principalmente en el primer momento, se refiere a competencias para contener y manejar la consternación y el desborde emocional de la población a la que intentarán ayudar. Pero también cuando ya ha pasado tiempo tras el desastre, si hubo cambios en las funciones por necesidad de responder a nuevas demandas del entorno, la incomodidad del trabajador puede sobrevenir tanto por tener que realizar tareas para las que se sienten sobrecalificados como porque no se sienten preparados, exponiéndose a la frustración y al fracaso (OPS, 2002, 2006).

- Ambigüedad en el rol. Producto de muchos de los factores anteriores, los espacios de rol de las personas pueden desdibujarse momentánea o más permanentemente: en el contenido del trabajo, en los parámetros de cumplimiento y de evaluación. Se produce incertidumbre, y de ella la presión por afrontarla y por adaptarse (GilMonte, 1999; OPS, 2002).

- Carencia de recompensas. No sólo de dinero o bonificaciones, sino más bien referido al reconocimiento de alguien (jefes, compañeros, la comunidad) de los esfuerzos adicionales y de la calidad del trabajo (Maslach, 2009).

Todo lo anterior produce una situación en la que los trabajadores deben desplegar afrontamientos eficaces para volverse competentes rápidamente. Si eso no ocurre, la persona enfrenta un escenario nuevo en el que puede sentirse incompetente, y en combinación con una vocación de servicios, impotente. Si a lo anterior se añade la imposibilidad de eludir o modificar el trabajo, puede desplegarse un escena- rio estresante porque no es tolerable pero tampoco tiene salida. Esta es la constelación de factores que considera la teoría explicativa del estrés llamada de "demanda-control", sustentada principalmente por Karasek desde la década de los 70's (en Cox, Griffiths y Rial-González, 2005; Karasek y Theore111990 en Maslach, 2009). Según ésta, un trabajo estresante sería más que un trabajo que produce sobredemanda: surge de una compleja interacción de factores situacionales del ambiente de trabajo, la cantidad de control que los individuos tienen sobre las demandas y su capacidad para responder a ellas (Caplan, 1998 citado en Parra, 2001).

\section{Sobrecargas en el trabajo emocional}

El trabajo emocional se define como el correlato de la interacción continua y por largos períodos entre el o la ocupante del rol laboral y los usuarios en áreas de protección social, salud, enfermedad y vulnerabilidad en general (Zapf, Seifert, Schmutte, Mertini, \& Holz, 2001). Es el tipo de trabajo que es usual en algunas ocupaciones y profesiones, como lo son trabajadores sociales, psicólogas/os y enfermeras/os, profesores y también gendarmes y policías. Se trata de puestos de trabajo que evocan un constante y significativo emocionar, a la vez que requiere manejo de la expresión y reacción frente a esas emociones (Zapf y cols., 2001).

Los requerimientos que hacen particular el trabajo emocional son básicamente tres: 1) una alta sensibilidad, que permita reconocer las emociones del usuario; 2) la capacidad de controlar la interacción, por ejemplo conteniendo y modulando la emoción en el otro con fines funcionales a la atención y a la ayuda; y 3) la capacidad de disonancia emocional, entendida como la habilidad de disimular ciertas emociones y conducirse o expresarse como si la emoción que se siente fuera otra, más funcional a las circunstancias (Zapf et al., 2001).

El trabajo emocional se vuelve más demandante en los escenarios de crisis. En ellos los trabajadores de emergencia, de salud y de asistencia social se enfrentan a situaciones extraordinarias porque es más extenso el sufrimiento de personas y comunidades completas. Respecto de las poblaciones que estaban en pobreza o vulnerabilidad antes del evento, es frecuente que a las dificultades que pudieran haber experimentado en torno a participar e integrarse a redes de sustento, se añadan tras los desastres mayores limitaciones de acceso a servicios de emergencia, salud o aumente el riesgo de sufrir los efectos de un trato discriminatorio en la oferta de ayuda (OPS, 2006). También los enfermos, los ni- 
ños pequeños y los adultos mayores pueden pasan a ser usuarios involuntarios de los sistemas de ayuda en estas circunstancias de mayor precariedad, y su sufrimiento suele causar cargas emocionales mayores (OPS, 2006).

Eso ofrece un contexto en que cada trabajador deberá atender a más usuarios y los usuarios vendrán a ellos con mayores carencias o apremios que lo usual, que constituye un flujo al que los trabajadores no estarían acostumbrados.

Estas condiciones exceden por mucho las circunstancias habituales de los trabajadores tanto cuantitativa como cualitativamente. Se suele asumir que estos profesionales están acostumbrados al dolor, las carencias o vulneraciones, pero es un error considerar que el volumen de carga emocional carece de relevancia (Zapf et al.; Bajoit, 2009).

Finalmente, otra ruta por la que el trabajo emocional se vuelve más demandante es porque en estas ocasiones desaparece el "efecto amortiguador" (buffer effect) que las redes sociales brindan para la contención y desahogo, necesario para encarar con éxito las demandas normales del trabajo emocional (López-Cabañas y Chacón, 1999).

Normalmente las personas que trabajan en auxilio de poblaciones vulnerables o vulneradas, cuentan con espacios familiares o de amistad para vaciarse, descargarse y recibir consuelo o consejo, tanto en casa como en el trabajo. En situaciones de crisis colectivas esos espacios pueden diluirse o desaparecer (OPS, 2002). Los cercanos pueden vivir sentimientos de desamparo y tristeza por sus propias pérdidas o fragilizaciones, por lo que las demandas de apoyo y contención se generalizan, dejando a estos trabajadores sin apoyos anteriores con los que contaban, o incluso siendo ellos demandados para asumir el rol de contención que se extiende, ahora, desde el trabajo a la casa o a los amigos.

En estas condiciones, los requerimientos de la comprensión, contención y control de las emociones propias y ajenas pueden constituir un factor de riesgo de patologías psicológicas, toda vez que el trabajador sienta que es imposible hacer frente individualmente a situaciones condicionadas organizacionalmente por la crisis. En esas circunstancias, el filtro y el control emocional pueden reducirse, por lo que el trabajador se expone a las indeseables consecuencias de la fatiga emocional y sus correlatos conductuales: desánimo, labilidad, irritabilidad, trato poco adecuado y descarga descontrolada de las emociones. Su desempeño laboral, así, puede verse severamente afectado.

\section{Modificaciones en las condiciones y recompensas habituales de la relación de ayuda}

Se ha denominado como relación de ayuda a aquella que se realiza cara a cara entre dos personas o grupos, una con necesidades y la otra con las competencias o conocimientos para ayudarle (Bajoit, 2009 , en imprenta). Se entiende como una relación asimétrica, justificada por la capacidad que tiene quien ayuda para otorgar asistencia al ayudado o para promover en el él/ellos el desarrollo y despliegue de recursos y potencialidades (Rogers, 1996).

Bajo situaciones especiales, esta relación puede transformarse en otro espacio adicional para experimentar los efectos negativos de condiciones de trabajo poco adecuadas, suscitadas por la emergencia y arrastradas tras meses del desastre. La caracterización propuesta por Guy Bajoit de las dimensiones de análisis de la relación de ayuda (2009, en imprenta) permite visualizar estos efectos:

La finalidad: la finalidad de toda relación de ayuda es ayudar a quien lo necesita, valga la redundancia. Pero en ocasiones como tras el terremoto, ese objetivo es alcanzado con dificultad o sencillamente no logra conseguirse. Las sobrecargas de la emergencia, el aumento en el número de personas en necesidad o la carencia de recursos para ofrecer soluciones hace que, en ocasiones, la finalidad de la relación de ayuda zozobre.

El deseo de dar solución a demandas que son apremiantes y legítimas, puede chocar con la incapacidad del sistema para responder. Resulta frecuente que los primeros días tras un desastre la capacidad organizacional de municipios o servicios de ayuda sean bajas por falta de personal, de respuestas, de recursos. En esas circunstancias la relación de ayuda resulta de riesgo para el trabajador porque puede ser fuente de sensaciones de incompetencia (no sé hacer más) e impotencia (no puedo hacer más pero me importa no poder hacerlo). De allí que finalmente, lo que cansa no es el trabajar para la gente, sino el no poder ayudarles (Pines, en Schaufeli, Maslach y Mareck, 1993).

Las contribuciones: como ya se dijo, pese a ser una relación asimétrica, es necesario que ambas partes de la relación de ayuda pongan una parte: quien ayuda pone soluciones, materializadas en cosas, conocimientos o servicios que debieran ser ofrecidas con deferencia y buen trato; quien es ayudado debe poner al servicio de la relación su capacidad para recibir pero también para facilitar el proceso de ayuda, y en un extremo deseable, para expresar 
genuina gratitud y reconfortar al trabajador en su anhelo de haber mejorado las condiciones de vida de quien acudió en busca de ayuda (Bajoit, 2009, en imprenta).

Cuando todo el sistema se recarga, las posibilidades de responder son bajas y la paciencia y comprensión del público van disminuyendo con el paso de las horas, por lo que la relación de ayuda puede esfumarse. La nueva relación se traduce en una relación de conflicto, de ataque y defensa y finalmente en una mala experiencia, que priva al trabajador de las recompensas y gratificaciones de su labor.

Las retribuciones y remuneraciones: esta dimensión puede analizarse en dos flancos. El primero dice relación con el hecho que, muchas veces, la mayor retribución que las relaciones de ayuda producen en los trabajadores es la percepción de trabajo bien hecho y de la meta propuesta lograda: ayudar a las personas y ver que mejoran es de por sí recompensante, que se traduce en un satisfactor intrínseco asociado al trabajo (Ferreiro y Alcazar, 2002). Pero este rasgo propio de las personas con vocación de servicio no impide que también aspiren a otra fuente de satisfacción, como es el reconocimiento (concreto o simbólico) y agradecimiento de jefes, compañeros y también de los usuarios, entendidos como satisfactores extrínsecos que reemplazan a otros que no suelen ser accesibles, como sueldos abultados, bonos o aumentos de salario (Ferreiro y Alcazar, 2002; Maslach, 2009).

En el caso del terremoto en Chile, se produjeron sobre esfuerzos y esfuerzos por parte del personal de emergencia, salud y ayuda tildados de heroicos entre las comunidades y los medios de comunicación. Algunos de esos gestos recibieron reconocimiento social en la prensa y televisión, y en ocasiones también por parte de los usuarios, jefes o compañeros de trabajo. Pero si eso no ocurrió, puede haberse producido frustración, puesto que la especial sensibilidad al reconocimiento social es un rasgo frecuente en las profesiones de ayuda (Bajoit, 2009, en imprenta; Maslach, 2009; OPS, 2002).

Explicado por medio de teorías organizacionales de la motivación, un desbalance negativo entre el aporte desplegado (se dio más de lo comprometido) y la recompensa percibida (se recibió menos de lo esperado) da como resultado la frustración en el trabajador (Lawler, 1983; Walster et al. 1973 y Siegrist 1996, en Maslach, 2009). Como acción correctiva, lo que puede ocurrir luego es que el trabajador responda al desbalance con una aportación menor que rectifique el desbalance percibido. Lo que se observará entonces es un trabajador que reduce su motivación, que disminuye su contribución, que aparece como incomprensible e injustificado cuando no se ha tenido en cuenta que su origen pudo estar en las ofertas de sentido y recompensa que la organización pone a disposición del trabajador.

\section{El impacto de las fuentes de riesgo post terremoto en la salud a través del rol de la identidad}

Tanto desde la perspectiva psicológica como sociológica, las organizaciones constituyen sistemas sociales conformados por roles relativamente estables, que enmarcan los puntos de referencia y observación de los trabajadores, así como las relaciones sociales que entablan desde ese espacio laboral (Cohen, 1998).

Los roles enmarcan no sólo comportamientos, sino espacios de experiencia con que las personas significan y observan lo que hacen, las interacciones con los usuarios, otros pares o con la organización, todo lo cual constituye una parte del quién soy. Con ello se hace énfasis en la idea que los roles son contextos sociales creados en las organizaciones que las personas viven como espacios de "realidad", desde los cuales se entiende y se narra una respuesta al quién soy laboralmente hablando, que para autores como McLean y Pasupathi, constituyen la identidad narrativa del trabajador (Hammack, 2008; McLean, Pasupathi, \& Pals, 2007; Pasupathi, Mansour \& Brubaker, 2007; Pasupathi, McLean y Weeks, 2009).

Desde este punto de vista se verá a la organización, al escenario que constituyen para el trabajador y a los factores psicosociales de riesgo y de protección como enmarcamientos organizacionales en los que la persona se definirá, se leerá a sí misma y a su rol. De allí que la consecuencia que puede derivarse tras un tiempo trabajando en unas malogradas condiciones laborales, es que los trabajadores concluyen que lo que han aportado vale poco (porque no tiene éxito reconocido), el objeto de su dedicación vale poco (la ayuda) o el puesto de trabajo vale poco (Pines, 1993 en Schaufeli, Maslach y Mareck, 1993). Esta conclusión puede producir un leve sinsabor o reacciones de sufrimiento más extremas. En éste último caso, desvalorizando su rol laboral, las personas terminan desvalorizándose también a sí mismos. Como bien señalaba Pines (1993), "los estresores organizacionales que implican condiciones insatisfactorias para trabajar no sólo estresan porque impiden que la persona se sienta competente y exitoso en lo que hace, sino porque envía el mensaje implícito que lo que hacen es insignificante" (Pines, 1993 en Schaufeli, Maslach y Mareck, 1993, p. 37). 


\section{Estrés, estresores y respuestas organizacionales}

Como ya se vio, definido al modo transaccional el estrés denomina una respuesta patológica en relación con la vivencia subjetiva de unas condiciones laborales sobredemandantes, que puede producir a corto y mediano plazo el síndrome de estrés agudo, y en el largo plazo y tras la configuración de estrés crónico, un potencial proceso de burnout. Desde esta interpretación resulta poco funcional intentar establecer quién debiera cargar con la responsabilidad de remediar las consecuencias que de ello se derivan. Esto contrasta con interpretaciones, vigentes aún hoy entre algunas jefaturas y organizaciones, donde el estrés se ve como un problema de debilidad individual y una carga que el trabajador "trae desde la casa", olvidando u obviando el hecho que la organización también determina situaciones que son contexto psicológico e identitario de las personas, y obviando que será la organización quien reciba las consecuencias en la salud de esas interacciones.

El asunto más bien debe observarse desde la cualidad de la experiencia más que desde la cantidad de influencia. Saber que el trabajador a vivido situaciones personales estresantes (en escenario donde no hay crisis adicionales) no es impedimento para que las instituciones asuman que ciertos factores organizativos contribuyen a dificultar el desempeño laboral al trabajador. Lo mismo cabe decir hoy, a cuatro meses del evento que remeció al centro-sur de Chile: el terremoto generó una serie de consecuencias, como ya se observaba en relación a deterioro físico, modificaciones de la organización del trabajo, de la cantidad de trabajo y sus efectos en la cuantía del trabajo emocional y en la relación de ayuda. Sin embargo, la organización tiene pese a todo un espacio insoslayable de acción que poner a disposición de los trabajadores, a partir de ahora si no lo ha hecho antes, para crear unas mejores condiciones para la recuperación de la calidad de vida laboral que su personal pudo haber perdido.

De acuerdo con lo anterior, las actividades de prevención y las que apuntan a facilitar la recuperación paulatina de la salud en el trabajo requiere de análisis y adquisición de competencias de todos los actores involucrado, con el fin de influir en algunas dimensiones organizacionales.

Reconocer que las reacciones de estrés agudo pueden ser normales frente a situaciones de crisis y cambio. Las organizaciones deben legitimar la experiencia de sufrimiento que sus trabajadores pueden estar vi- viendo, sea en privado o en público, aún cuando se crea (erróneamente) que ya ha pasado mucho tiempo. La literatura especializada reconoce que muchos de los efectos acumulados más perniciosos para la salud pueden comenzar a evidenciarse tras seis meses del evento crítico (OPS, 2006). Si en las organizaciones las jefaturas asumen que ya todo volvió a lo de siempre y desnormalizan o descontextualizan las reacciones perdurables de estrés laboral, aislarán al trabajador en una interpretación que responsabiliza únicamente a la persona de su padecer, dando comienzo a una experiencia subjetiva peor de la que venía forjándose. Es necesario el reconocimiento de la organización de la realidad y normalidad de las reacciones de estrés que tienen su punto de partida (y quizá de llegada) en el desastre natural vivido.

Adicionalmente resulta beneficioso crear entornos donde se reconoce explícitamente que las alternativas de reacción van desde la sana adaptación hasta las reacciones patológicas. Sólo así se creará el clima de confianza y respeto que hace falta para abrirse a interpretaciones y significaciones menos dañinas de la experiencia (no soy el único, no soy un raro/a o un débil). Recuérdese que el estrés es el correlato psicológico de sentirse en sobrecarga, ansiedad y desesperación, por lo que si el contexto acepta y legitima esas reacciones como normales, se sientan las bases para una evaluación individual más benigna, menos estresante.

Reconocer el trabajo realizado en esas condiciones y no sólo el trabajo extraordinario. Pese a que felicitar y realizar un reconocimiento del trabajo bien hecho tiene más efecto en el momento mismo del desempeño, nunca es tarde para realizar actos de refuerzo y agradecimiento por lo que estos profesionales de ayuda hicieron por sus comunidades, pese a sus propios miedos y pérdidas en ese momento. Estos profesionales trabajan en ámbitos que normalmente tiene escaso estatus social, salarios poco atractivos y pese a ello encuentran compensación en el servicio que prestan a otros. De allí que sean sensibles, como se dijo, a las muestras de gratitud o de visibilización de los esfuerzos que desplegaron o han desplegado, principalmente el que viene de las jefaturas (GilMonte, 1999; Maslach, 2009, OPS, 2002). Si fuera posible realizar compensaciones concretas o pecuniarias aún mejor, pero al menos debieran hacerse gestos socialmente significativos, que además provengan de la institucionalidad de la organización y no sólo como gestos independientes e individuales de algunas jefaturas.

Aumentar capacidades individuales, grupales y organizacionales. Cuando una situación desborda la 
capacidad de respuesta adaptativa de la población, se necesita aumentar y diversificar las herramientas de las personas de afrontar esos hechos. Tras el reconocimiento es importante que las organizaciones provean a sus trabajadores de capacitación y entrenamiento en el desarrollo de estrategias de afrontamiento, tanto individuales como grupales, para hacer frente a las fuentes de estrés, pero también para que puedan incorporar en forma integrada y positiva la nueva normalidad. Nada más dañino que intentar, tras pocos meses de ocurrido el terremoto, discursos de "aquí no ha pasado nada" o de "ya hemos vuelto a la normalidad" porque, en estricto rigor un desastre es algo tras lo cual nunca nada vuelve a ser como era. Aceptar los puntos de no retorno y aprender a cambiar aquello que aún se pueda (y distinguir entre lo uno y lo otro, como reza el dicho) son los primeros pasos de una adaptación psicológica madura y exitosa. Y también los pasos que daría una organización saludable y proectora de sus trabajadores.

Cursos de capacitación que aumenten el repertorio de afrontamiento emotivo, cognitivo y conductual de los trabajadores y jefaturas; entrenamiento en el reconocimiento de factores protectores y estresores psicosociales presentes y finalmente la manera de actuar frente a ellos, fundan las bases de una mayor capacidad organizacional para aprovechar los recursos y oportunidades que pueden ponerse a disposición del cuidado de la salud ocupacional.

Re-enmarcar los nuevos roles y referencias. Cuando el cambio en las condiciones en que se desarrolla el trabajo modifican el rol, las fuentes de recompensa o la posición social relativa, se produce un alto grado de incertidumbre respecto de qué debo hacer y de quién soy laboralmente hablando, que pueden transformarse en un elemento que predisponga efectos psicológicos negativos (Cohen, 1998; GilMonte, 1999).

Una manera de evitar el potencial de daño de este aspecto sobre la experiencia del trabajador es enmarcar organizacionalmente ese cambio lo antes posible en al menos cinco dimensiones: (1) en el tiempo (cuánto tiempo durará); (2) en su evolución (qué situación viene después); (3) recompensas concretas y simbólicas (incentivos u oportunidades derivadas del cambio de situación); (4) posición relativa en torno a otros (si hubo cambio en jefatura a la que reportar, en quién se considera par o compañero de equipo o en torno a los subalternos) y (5) Nuevas metas o resultados que se esperan de sus gestiones en el trabajo (para evitar la sensación de fracaso que puede sobrevenir a una mala compren- sión de las expectativas que se tienen de sus logros y de su puesto). El proveer certidumbres en al menos estas dimensiones es el rol del liderazgo en las organizaciones, por lo que resulta adecuado ejercerlo para disminuir una fuente de estrés en el trabajo.

Diagnóstico de necesidades especiales. Frente a las dudas personales respecto del grado de normalidad o patología psicológica de una persona, lo indicado es realizar diagnósticos profesionales. Esto, con el fin de evitar los sesgos optimistas (no debo estar tan mal o ya se me va a pasar sólo) o pesimistas (no saco nada con hacer algo, nada va a mejorar) en situación de aislamiento en el que no puede desconfirmarse ninguna de los dos sesgos.

La gran mayoría de las personas pueden encarar los procesos de adaptación de manera autónoma, con el apoyo y ayuda de familiares, amigos, compañeros. Sin embargo, si tras pasar los meses la persona no se siente conforme con su estado de salud o con la calidad de su entrega en el trabajo, la familia o la comunidad (porque sentirse como antes no resulta realista), es de suma importancia realizar un diagnóstico y recibir prescripción de tratamiento.

Supervisión atenta. Es sabido que las jefaturas cumplen una función relevante en torno a la gratificación y realización en el trabajo, puesto que son quienes controlan el acceso a satisfactores de diverso origen, pero también porque pueden restringir el acceso del trabajador hacia ellos (Ferreriro y Alcazar, 2002). Dentro de los satisfactores intrínsecos el explicitar el valor del trabajo desempeñado por el trabajador, en todo aquello que se pueda valorar, funciona como fuente de retroalimentación positiva y de recompensa simbólica. Al contrario, la falta de reconocimiento se lee como indiferencia y minusvaloración del desempeño alcanzado.

En lo extrínseco, el ofrecer condiciones de trabajo que señalen la dignidad y relevancia del trabajo también resulta protector para el trabajador. Si bien en ocasiones se trata de un problema de disponibilidad de recursos, en otras requiere más bien de intencionar la administración de recursos y eliminar fuentes evitables de estrés del entorno físico que deben comprenderse como inaceptables.

Otro aspecto al que debe prestar atención una supervisión intencionada para mejorar la adaptación al trabajo tras el terremoto es a las secuelas psicológicas y su correlato conductual. Las reacciones al estrés, al miedo, la tristeza y al mismo burnout pueden verse diferente en las personas: es más frecuente que las mujeres lloren frente a la tristeza, pero los hombres se vuelven más irascibles bajo su influjo; la despersonalización en el trato que apa- 
rece en unos como humor negro y sarcasmo hacia el usuario, en otros aparece como frialdad e indiferencia. Asimismo, las tensiones en las relaciones de equipo, el aislamiento y la elusión de contacto con compañeros y usuarios que antes solía producir placer y bienestar pueden señalar estados de ensimismamiento defensivo frente a las características aversivas en el ambiente laboral.

Este darse cuenta de las jefaturas permite diseñar, con participación del trabajador, planes de afrontamiento orientados a favorecer la paulatina adaptación a las funciones y roles, pero también la adecuación del trabajo al trabajador. Éstas intervenciones deben estar orientadas por evidencia empíricamente validada, y diseñadas para responder a factores psicosociales específicos detectados en diagnósticos llevados a cabo con tal objetivo (Vega, 2009).

\section{A modo de conclusión}

Era deseo en este artículo mostrar la faceta en que la organización constituye un espacio de realidad psicológica relevante para los trabajadores, y cómo ese espacio puede volverse, tras desastres como el vivido en Chile, una realidad agobiante. Bajo las definiciones utilizadas de estrés y burnout como patologías de origen ocupacional, las organizaciones se ven interpeladas en su doble rol de prevenir la influencia de factores psicosociales de riesgo pero también de promover la salud, a través de la gestión cotidiana de las personas, de sus roles, de los recursos físicos, de las reglas y de la institucionalización de modos de funcionamiento que estén a la altura de la magnitud del estresor que se impuso para todos tras el 27 de Febrero.

Las ocupaciones y profesiones de ayuda son tanto una necesidad social de primer orden como un una vocación y líneas de un relato al interior de la identidad. Y es probable que sigan siéndolo. Es por ello que encuentra sentido y justificación una apelación a esas organizaciones de ayuda, de salud, de emergencia y de seguridad ciudadana por involucrarse activamente en poner a disposición de sus trabajadores los incentivos, psicológicos y materiales, que alimenten de gratificaciones esos relatos y experiencias.

Finalmente, y en una perspectiva de promoción de la salud, es de importancia permitir al trabajador hacerse dueño de sus circunstancias, entendiendo que el trabajo y la organización como sistemas sociales también son una realidad de las que ellos participan. En nombre de esa perspectiva, cualquier desarrollo y apuesta técnicamente bien hecha tendrá el efecto de aumentar la capacidad de la organización para encarar su misión con éxito, pese a los ineludibles efectos de los desastres naturales que periódicamente desafían la capacidad de asombro, de resistencia y de adaptación.

\section{Bibliografía}

BAJOIT, G. (2009). Pour une sociologie de combat. En imprenta.

BRYCE, C. (2001). Isights into the concepts of stress. Washington D.C.: Pan American Health Organization.

CAPLAN, G. (1964). Principles of preventive psychiatry. New York: Basic Books, 1964.

COHEN, R. (1998). Salud mental para víctimas de desastres. Mexico: Organización Panamericana de la Salud.

COX, T., GRIFFITHS, A. y RIAL-GONZÁLEZ, E. (2005) Investigación sobre el estrés relacionado con el trabajo. Luxemburgo: Agencia Europea para la Seguridad y la Salud en Trabajo.

FERREIRO, P. y ALCÁZAR, M. (2002). Gobierno de personas en la empresa. España: Editorial ARIEL.

FREUDENBERGER, H. (1974). Staff burn-out. Journal of Social Issues, Vol. 30, № 1, 1974, pp. 159-165.

GIL-MONTE, P. (2003). El síndrome de quemarse por el trabajo (síndrome de burnout) en profesionales de enfermería. Ponencia presentada en el I Seminario Internacional sobre Estrésse e Burnout, Curitiba (Brasil), en agosto de 2002

GIL-MONTE, P. y PEIRÓ, J. (1999). Perspectivas teóricas y modelos interpretativos para el estudio del síndrome de quemarse por el trabajo. En Anales de Psicología, año 1999, Vol 15, № 002, pp. 261-268.

HAMMACK, P. (2008). Narrative and the cultural psychology of identity. Personality and social psuchology review, 2008; Vol. 12, №3; pp. 222-247.

LÓPEZ-CABAÑAS, M. y CHACÓN, F. (1999). Intervención psicosocial y servicios sociales: en enfoque participativo. España: Ed. Síntesis.

MASLACH, C. (2009). Comprendiendo el burnout. Ciencia y Trabajo, abril-junio, 2009, 11 (32), pp. 37-43.

MASLACH, C., SCHAUFELI, W. y LEITER, M. (2001). Job burnout. Annual Review of Psychology; 2001; 52, pp. 397-422.

MASLACH, C. y JACKSON, S. (1981). The measurement of experienced burnout. Journal of Occupational Behaviour, Vol. 2, №2 (April, 1981), pp. 99-113.

MCLEAN, K., PASUPATHI, M. y PALS, J. (2007). Selves creating stories creating selves: a process model of selfdevelopment. Personality and Social Psychology Review, Vol. $11 \mathrm{~N}^{\circ} 3$, August 2007, pp. 262-278. 
OLIVARES, V. y GIL-MONTE, P. (2007). Prevalencia del síndrome de quemarse por el trabajo (burnout) en trabajadores de servicios de Chile. En Información Psicológica, septiembre 07-diciembre 07, pp. 43-52.

OLIVEIRA, L., TENORIO, J., DE SOUZA, A., PEREIRA, E. y SOUZADA, W. (2002). A síndrome de burnout e os valores organizacionais: Um estudo comparativo em hospitais universitarios. En Psicologia: reflexao e critica, Vol. 15, número 1, Universidad Federal do Río Grande do sul, Puerto Alegre, Brasil, pp. 189-200.

ORGANIZACIÓN PANAMERICANA DE LA SALUD (2002). Protección de la salud mental en situaciones de desastres y emergencias. Washington DC: Organización Panamericana de la Salud.

PARRA, M. (2003). Conceptos básicos en salud laboral. Santiago: Oficina Internacional del Trabajo.

PARRA, M. (2001). Salud mental y trabajo. Santiago de Chile: Monografías de gestión en psiquiatría y salud mental, Universidad de Santiago.

PASUPATHI, M., MANSOUR, E., y BRUBAKER, J. (2007). Developing a Life Story: Constructing relations between self and experience in autobiographical narratives. Human Development, 50 (2/3), 85-110.

PASUPATHI, M., MCLEAN, K. y WEEKS, T. (2009). To Tell or Not to Tell: Disclosure and the Narrative Self. Journal of Personality 77:1, February 2009, pp. 89-124.

PINES, AYALA. (1993). Burnout: an existencial perspective. En Schaufeli, W., Maslasch, C. y Marek, T. (1993). Professional burnout: recent developments in theory and research. Washington: Taylor \& Francis ( $1^{\circ}$ edición).

RODRÍGUEZ, J. (editor). (2006).Guía práctica de salud mental en situaciones de desastres. Washington D.C.: Organización Panamericana de la Salud.

SCHAUFELI, W., MASLACH, C. y MAREK, T. (editores) y (1993). Professional burnout: recent developments in theory and research. Washington, D.C.: Taylor \& Francis.

SONNENTAG, S. (2005). Burnout research: Adding an off-work and day-level perspective. En Work \& Stress, July-September 2005; 19(3): 271-275.

TARIS, T., LE BLANC, P., SCHAUFELI, W., SCHREURS, P. (2005). Are there causal relationships between the dimensions of the Maslach Burnout Inventory? A review and two longitudinal tests. En Work \& Stress, July-September 2005; 19(3): 238-255.

TRUCCO, M., VALENZUELA, P. y TRUCCO, D. (1999). Estrés ocupacional en personal de salud. Revista médica de Chile, v.127 n.12.

VEGA, S. (coordinadora) y (2009). Experiencias en Intervención Psicosocial. Más allá de la evaluación del riesgo. España: Ministerio de trabajo y migración-Instituto nacional de seguridad e higiene en el trabajo.
ZAPF, D., SEIFERT, C., SCHMUTTE, B., MERTINI, H., HOLZ, M. (2001). Emotion work and job stressors and their effect on burnout. Psychological Health, 16, pp. 527-545, 2001. 